\title{
Recruitment of cognitive control regions during effortful self- control is associated with altered brain activity in control and reward systems in dieters during subsequent exposure to food commercials
}

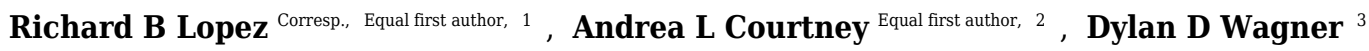 \\ 1 Department of Psychological Sciences, Rice University, Houston, Texas, United States \\ 2 Department of Psychology, Stanford University, Stanford, California, United States \\ 3 Department of Psychology, The Ohio State University, Columbus, Ohio, United States \\ Corresponding Author: Richard B Lopez \\ Email address: richard.lopez@rice.edu
}

Engaging in effortful self-control can sometimes impair people's ability to resist subsequent temptations. Existing research has shown that when chronic dieters' selfregulatory capacity is challenged by prior exertion of effort, they demonstrate disinhibited eating and altered patterns of brain activity when exposed to food cues. However, the relationship between brain activity during self-control exertion and subsequent food cue exposure remains unclear. In the present study, we investigated whether individual differences in recruitment of cognitive control regions during a difficult response inhibition task are associated with a failure to regulate neural responses to rewarding food cues in a subsequent task in a cohort of 27 female dieters. During self-control exertion, participants recruited regions commonly associated with inhibitory control, including dorsolateral prefrontal cortex (DLPFC). Those dieters with higher DLPFC activity during the initial selfcontrol task showed an altered balance of food cue elicited activity in regions associated with reward and self-control, namely: greater reward-related activity and less recruitment of the frontoparietal control network. These findings suggest that some dieters may be more susceptible to the effects of self-control exertion than others and, whether due to limited capacity or changes in motivation, these dieters subsequently fail to engage control regions that may otherwise modulate activity associated with craving and reward. 
RECRUITMENT OF COGNITIVE CONTROL REGIONS DURING EFFORTFUL SELF-CONTROL IS ASSOCIATED WITH ALTERED BRAIN ACTIVITY IN CONTROL AND REWARD SYSTEMS IN DIETERS DURING SUBSEQUENT EXPOSURE TO FOOD COMMERCIALS

Richard B. Lopez ${ }^{*}$, Andrea L. Courtney², Dylan D. Wagner ${ }^{3}$

${ }^{1}$ Department of Psychological Sciences, Rice University, Houston, TX 77005, USA

${ }^{2}$ Department of Psychology, Stanford University, Stanford, CA 94305, USA

${ }^{3}$ Department of Psychology, The Ohio State University, Columbus, OH 43210, USA

Corresponding Author:

Richard Lopez

6500 Main Street, MS-663, Houston, TX 77030, USA

Email address: richard.lopez@rice.edu 
42

43

44

45

46

47

54

\section{ABSTRACT}

Engaging in effortful self-control can sometimes impair people's ability to resist subsequent temptations. Existing research has shown that when chronic dieters' self-regulatory capacity is challenged by prior exertion of effort, they demonstrate disinhibited eating and altered patterns of brain activity when exposed to food cues. However, the relationship between brain activity during self-control exertion and subsequent food cue exposure remains unclear. In the present study, we investigated whether individual differences in recruitment of cognitive control regions during a difficult response inhibition task are associated with a failure to regulate neural responses to rewarding food cues in a subsequent task in a cohort of 27 female dieters. During self-control exertion, participants recruited regions commonly associated with inhibitory control, including dorsolateral prefrontal cortex (DLPFC). Those dieters with higher DLPFC activity during the initial self-control task showed an altered balance of food cue elicited activity in regions associated with reward and self-control, namely: greater reward-related activity and less recruitment of the frontoparietal control network. These findings suggest that some dieters may be more susceptible to the effects of self-control exertion than others and, whether due to limited capacity or changes in motivation, these dieters subsequently fail to engage control regions that may otherwise modulate activity associated with craving and reward. 
70

\section{INTRODUCTION}

72

73

74 75 Heatherton, 2000). 94 phenomena.

Broadly defined, self-regulation refers to the human capacity to flexibly regulate

thoughts, emotions, and behaviors. Self-control, in particular, describes those situations in which self-regulatory processes are engaged to inhibit prepotent or automatic impulses, especially when these impulses conflict with other goals (Carver, 2005; Heatherton \& Wagner, 2011; Hofmann, Friese, \& Strack, 2009). In situations when impulses and cravings are relatively weak, less effort is required to engage in self-control. However, at other times, self-control can be experienced as effortful. A large body of research in experimental psychology has examined how the effects of exerting self-control in one domain can lead to self-regulation failure in other domains, resulting in the hypothesis that self-regulation is dependent on limited cognitive resources that can become depleted over subsequent self-regulation attempts (Baumeister, Bratslavsky, Muraven, \& Tice, 1998; Baumeister, Vohs, \& Tice, 2007; Muraven, Tice, \& Baumeister, 1998; Vohs \&

5 Most recently, the consensus appears to be that the available evidence, in favor of and/or against self-regulatory depletion effects, is inconclusive (Friese, Loschelder, Gieseler, Frankenbach, \& Inzlicht, 2018). However, a recent pre-registered study with large samples (Ns $\geq$ 377) has shown reliable effects consistent with an attentional-based account of depletion (Garrison, Finley, \& Schmeichel, 2018), and studies using more ecologically valid designs have provided supporting evidence for the role of self-regulatory fatigue in precipitating self-control

1 failures in daily life (Hofmann, Baumeister, Förster, \& Vohs, 2012; Wilkowski, Ferguson,

2 Williamson, \& Lappi, 2018), suggesting, perhaps, that focusing on more ecologically valid and 3 appetitive behaviors (such as dieting failures) may provide better traction on the underlying 
96 dieters (i.e., those who constantly monitor and attempt to control their food intake) as they

97 frequently demonstrate weight fluctuations, weight gain, and self-control lapses (Heatherton,

98 Polivy, \& Herman, 1991; Lowe et al., 2006; Lowe, Doshi, Katterman, \& Feig, 2013), including

99 after effortful self-control exertion (Friese, Engeler, \& Florack, 2015; Kahan, Polivy, \& Herman,

100 2003; Vohs \& Heatherton, 2000). Much of this prior work has generally treated dieters as a

101 homogenous group, comparing these to non-dieters or to groups of dieters who were not

102 "fatigued" by self-control exertion (e.g. Wagner et al. 2013). However, more recent work has

103 taken an individual differences approach to better understand why some dieters may be more

104 prone to self-control failure than others. For example, prior work in our lab has shown that those

105 dieters who experience weaker food cravings and more positive mood in daily life are also most

106 successful in controlling impulses to eat (Lopez, Milyavskaya, Hofmann, \& Heatherton, 2016).

107 Another study revealed that after initial exertion of (effortful) self-control, dieters variably

108 recruited brain regions associated with self-control and reward when exposed to appetizing food

109 cues, and these differences predicted self-control outcomes in daily life (Lopez, Chen, Huckins,

110 Hofmann, Kelley, \& Heatherton, 2017). However, this study did not measure brain activity

111 during the initial self-control task. So, a key unanswered question is: are individual differences in

112 recruitment of self-control brain regions during self-control exertion in dieters related to altered

113 patterns of activity in brain regions associated with control and reward when dieters are

114 subsequently exposed to appetizing food cues? Additionally, might a relationship between brain

115 activity during self-control exertion and later exposure to tempting food cues itself predict self-

116 regulation failures (i.e., overeating) in more ecologically valid settings? 
118 examine individual differences in the neural correlates of effortful self-control among chronic

119 dieters and related activation in prefrontal regions associated with response inhibition to

120 subsequent recruitment of control and reward-related brain areas during exposure to appetitive

121 food commercials. In addition to their constant deployment of self-control, the dieting population

122 is characterized by a motivation to regulate food intake, allowing for cleaner operationalization

123 of self-control success and failure. In a recent brain imaging study, dieters randomly assigned to

124 first complete an effortful self-control task showed, on average, significantly higher activity in

125 orbitofrontal cortex (Haber \& Knutson, 2010; OFC; a key region in the brain's reward system;

126 O’Doherty, 2004; Suzuki, Cross, \& O’Doherty, 2017) during subsequent exposure to food cues,

127 as well as reduced functional coupling between inferior frontal gyrus (IFG), an area of lateral

128 prefrontal cortex, and the $\mathrm{OFC}$ - relative to dieters assigned to the control condition (Wagner,

129 Altman, Boswell, Kelley, \& Heatherton, 2013). These findings indicated that when dieters

130 engage in effortful self-control, the reward value of food may become amplified while self-

131 control capacity — as indexed by reduced functional connectivity between OFC and IFG—may

132 be compromised.

133 Work in cognitive neuroscience has identified lateral prefrontal cortex as a robust neural

134 correlate of self-control, indexing one's capacity to engage in response inhibition (see Aron,

135 Robbins, \& Poldrack, 2014 for a review). Lateral prefrontal cortex has also been implicated in

136 successful resistance of desires to smoke cigarettes as well as regulate food cravings in daily life

137 (Berkman, Falk, \& Lieberman, 2011; Lopez, Hofmann, Wagner, Kelley, \& Heatherton, 2014).

138 Additionally, several studies have found a general pattern such that, following initial exertion of

139 self-control, activity in prefrontal cortex subsequently decreases on subsequent tasks (Friese, 
140 Binder, Luechinger, Boesiger, \& Rasch, 2013; Hedgcock, Vohs, \& Rao, 2012; Luethi et al.,

141 2016; Persson, Larsson, \& Reuter-Lorenz, 2013) and the magnitude of this decrease has been

142 found to be correlated with performance deficits in subsequent cognitive tasks (Friese et al.,

143 2013; Persson et al., 2013). Moreover, these effects may be most pronounced in contexts where

144 people are required to inhibit responses to stimuli with high reward value (Freeman \& Aron, 145 2016).

146 Given these previous lines of work on self-control among dieters and the role prefrontal

147 cortex plays in effortful self-control in the cognitive domain, we hypothesized that individual

148 differences in dieters' recruitment of lateral prefrontal cortex during initial self-control exertion

149 would be associated with: (1) altered brain responses during subsequent exposure to food

150 commercials, and (2) ad libitum eating patterns following a later diet breaking episode. To test

151 these hypotheses, we first measured brain activity as dieters performed an effortful self-control

152 task in which they were required to actively inhibit reading a series of words that appeared on the

153 screen over the course of seven minutes. Following this, participants engaged in a food-cue

154 reactivity task involving food commercials that has previously been shown to reliably recruit the

155 brain's reward system (Rapuano et al., 2016). This dual-task design allowed us to examine the

156 correspondence between task-elicited activity in the lateral prefrontal cortex during self-control

157 exertion in the first task and the balance between regions associated with self-control and those

158 associated with food-reward in the second task. Here, we focus specifically on the relative

159 balance of activity in regions associated with self-control and reward during exposure to food

160 commercials, as our previous work has associated this balance measure with failure to resist the

161 desire to eat in daily life, as measured by experience sampling (Lopez et al., 2017). As some

162 have recently argued that self-control capacity may be better captured by the coordination of 
163 whole brain systems that support regulatory processes (e.g., frontoparietal control network),

164 rather than activity of discrete, independent regions (Kelley, Wagner, \& Heatherton, 2015), this

165 balance measure was calculated using a systems-based approach. Following the procedure used

166 previously in Lopez and colleagues (2017), we used independently defined, a priori

167 regions/systems of interest, namely the frontoparietal control network, which enables flexible

168 exertion of self-control (Power et al., 2011), and key regions of the reward system, namely OFC

169 and ventral striatum, both of which reliably activate to appetizing food images (Courtney,

170 Rapuano, Sargent, Heatherton, \& Kelley, 2018; Demos, Heatherton, \& Kelley, 2012; Rapuano,

171 Huckins, Sargent, Heatherton, \& Kelley, 2015; Rapuano et al., 2016; Wagner et al., 2013).

172

To constrain our hypotheses about potential relationships between PFC activity during

173 effortful self-control and subsequent recruitment of control (vs. reward) regions during food cue

174 exposure, we based our hypotheses on prior neuroimaging studies (Luethi et al., 2016; Persson et

175 al., 2013) that suggest that following (effortful) exertion of self-control, there is less recruitment

176 of lateral prefrontal cortex, compared to control groups that did not exert self-control, in a

177 subsequent task requiring self-regulation. Thus, in line with this work we focused on individual

178 differences among a group of dieters and hypothesized that dieters showing more recruitment of

179 prefrontal cortex during effortful self-control, indexing task difficulty and/or the experience of

180 effort, would subsequently show less recruitment of the frontoparietal control network and

181 therefore more reward-related activity in OFC and VS when viewing food commercials - thus

182 serving as evidence of a failure to appropriately engage self-control systems when confronted

183 with appetitive food stimuli. Finally, we also hypothesized that higher PFC activity during

184 effortful self-control, and/or lower frontoparietal (vs. reward) balance scores, would be 
185

186

187

188

189

190

191

192

193

194

195

196

197

198

199

200

201

202

203

204

205

206

207

208

209

associated with more disinhibited eating following a diet breaking episode — as measured in separate experimental session with the same participants.

\section{MATERIALS \& METHODS}

\section{Participants}

Thirty-two female dieters $\left(\mathrm{M}_{\mathrm{age}}=19.48, \mathrm{SD}_{\mathrm{age}}=1.11\right)$ were recruited from the Dartmouth community to participate in a two-part neuroimaging study for course credit. Dieting status was assessed by the Restrained Eating Scale (Heatherton, Herman, Polivy, King, \& McGree, 1988; Herman \& Polivy, 1980), and all participants were prescreened to ensure that they were actively dieting at the time the study was conducted. The study consisted of an initial fMRI scanning session in which brain activity was measured both during an effortful self-control task (Wagner et al., 2013) and a food cue reactivity task that used naturalistic food stimuli (i.e., fast food commercials; Rapuano et al., 2015). Approximately a week later, participants returned to the lab for a follow-up behavioral session, in which their diets were broken and subsequent disinhibited eating was measured, as per a previously validated used diet-breaking procedure (Demos, Kelley, \& Heatherton, 2011; Timko, Juarascio, \& Chowansky, 2012; Tomiyama, Moskovich, Haltom, Ju, \& Mann, 2009). All participants gave informed consent in accordance with guidelines set by the Committee for the Protection of Human Subjects at Dartmouth College, and were fully debriefed at the end of the study (IRB approval \#20325).

\section{Procedure}

In order to ensure participants remained naïve to the goals of the experiment, they were instructed that the study was primarily about attention and perception. Specifically, the experimenter told each participant the following: "We're interested in the relationship between attention and perception. Specifically, we will be exploring multiple types of perceptual processes, from higher-level person perception to lower-level sensory perception. Today we will 
210 be scanning your brain during an attention task, which will be followed by an episode of the 211 popular TV show Big Bang Theory, as you would see it on TV with commercials. We are

212 interested in how you perceive these popular TV characters. During the second visit, we'll have 213 you do some tests of lower level perception involving different senses, such as vision, sight, 214 smell, and taste."

215 Participants first completed an effortful self-control task adapted from Wagner and 216 colleagues' (2013) study that was modified to be amenable to neuroimaging analysis (i.e., 217 jittered presentation of stimuli and explicit baseline periods were added). For this task, all 218 participants were told that they would watch a clip from a nature documentary featuring 219 Canadian bighorn sheep, and at times various words would appear and move around on the 220 screen, but they were told to "avoid reading these distractor words whenever they appear." On 221 average, dieters find this task significantly more effortful, compared to a control condition in 222 which they just watched the film and read the words as they pleased, Cohen's $d=1.04$ 223 (calculated from Wagner et al., 2013). The video lasted for seven minutes, with a jittered 224 presentation of 40 words (all one-syllable and of neutral valence, e.g., "CHAIR" and "BOOK") 225 throughout the task epoch. Following the effortful self-control task, participants watched a complete episode of the 227 popular television sitcom The Big Bang Theory. In between episode segments, participants 228 viewed sets of commercials, with a total of 12 food commercials and 12 non-food (control) 229 commercials that were matched for duration and level of engagement and interest (see Figure 1 230 in Rapuano et al., 2015 for a depiction of the design). Food commercials featured menu items at popular fast food restaurants, such as

232 McDonald's, Wendy's, and Kentucky Fried Chicken. In contrast, control commercials featured 
233 various products that were not directly consumptive or appetitive (e.g., 4G LTE cellular service, 234 cars, cleaning products, shaving supplies). At the conclusion of the scanning session, we asked

235 participants to report on their experiences of the effortful self-control control task. Specifically, 236 they provided ratings on a 1-7 scale in response to questions that asked: "How difficult did you 237 find this task?" and "How much did this task 'tire you out' or make you feel mentally 238 exhausted/fatigued afterwards?"

Approximately one week after the scanning session, participants returned to the lab for a 240 follow-up behavioral session, consisting of a commonly used milkshake preload manipulation 241 (Brace, Crombag, \& Yeomans, 2016; designed to temporarily break participants diets; Demos et 242 al., 2011; Herman, Polivy, \& Esses, 1987; Mills \& Palandra, 2008) followed by ad libitum, 243 disinhibited eating of ice-cream. After drinking an entire 15-ounce milkshake, participants were 244 instructed to sample three flavors of ice cream under the guise of a taste test. Previous studies 245 have demonstrated that dieters whose diets are broken tend to eat more ice cream, but there are 246 individual differences in amount of ice cream consumed (Demos et al., 2011). The total amount 247 (grams) of ice cream consumed was measured using an Ozeri Pro Digital Kitchen Food Scale 248 (Ozeri USA, San Diego, CA). Previous research using this laboratory-based manipulation has 249 consistently demonstrated that following a milkshake preload, dieters will proceed to eat 250 significantly more ice cream than those participants whose diets are not broken (e.g., Demos et 251 al., 2011; Heatherton \& Baumeister, 1991; Herman \& Mack, 1975; Tomiyama et al., 2009).

\section{Analysis of fMRI data}

All analyses of neuroimaging data were conducted using SPM8 (Wellcome Department of Cognitive Neurology, London, England), along with tools for batch preprocessing and analysis (available at http://github.com/ddwagner/SPM8w). First, we carried out standard 
256 preprocessing procedures for both inhibitory control and cue reactivity tasks, to account for

257 motion related artifact (by including six motion regressors in first-level GLM model

258 specifications) and increase images' signal-to-noise ratio via spatial smoothing (8-mm FWHM

259 kernel). Between the two tasks, five participants' data were excluded due to excessive motion,

260 defined as $\geq 2$ instances of $\geq 2$ millimeters of movement in the $\mathrm{x}, \mathrm{y}$, or $\mathrm{z}$ plane (resulting $\mathrm{N}=27$

261 for subsequent analysis). In the case of the main analysis (relating DLPFC activity to subsequent

262 food cue reactivity), there were 26 participants who had complete data across those two

263 measures. So, for those reported analyses, $\mathrm{N}=26$.

264 Brain data for the two tasks were separately modeled and analyzed. First, for the effortful

265 self-control task, we ran a whole-brain univariate GLM analysis at the first (subject) level, with

266 two regressors specifying when words appeared on the screen and when words were absent (i.e.,

267 only the film was playing). The main contrast of interest (words present vs. absent) identified

268 period of the task when participants were actively engaging in self-control. Group-level T-maps

269 were then generated using random effects analysis of all subjects' word present-versus-word

270 absent contrast images. To correct for multiple comparisons, we performed cluster-based

271 correction via the AFNI tool $3 d$ ClustSim, which performed 10,000 simulations using the mean

272 smoothness estimated from residual images (obtained from each participant's first-level GLM)

273 and using spatial autocorrelation function parameters (via 3dClustSim's "acf" option) — as per

274 recent recommendations (Cox, Chen, Glen, Reynolds, \& Taylor, 2017). These simulations

275 returned a minimum cluster size of 25 voxels at an uncorrected $p<.001$ threshold required for a

276 cluster-level false positive discovery rate of $p<.05$ (thresholded map available at:

277 https://neurovault.org/collections/EDTWSFKM/images/60660/). 
280 the urge to read words whenever they appeared on the screen. This map revealed activity in 281 regions of both prefrontal and parietal cortex associated with attention and self-control (see 282 Figure 1 for thresholded T-map, indicated by orange/yellow gradient; Table 1 for a full list of 283 supra-threshold regions of peak activity). To test our hypothesis that lateral prefrontal cortex 284 would be engaged during effortful self-control, we extracted parameter estimates from the cluster 285 in the dorsolateral prefrontal cortex (peak MNI coordinates $X=30, Y=42, Z=24$ ) using a spherical 286 ROI with a 6-millimeter radius. Additionally, given the role the frontoparietal network plays in 287 cognitive control generally, and self-regulation of eating more specifically (Lopez et al., 2017), 288 we extracted standardized, aggregate activity across 8 nodes of the frontoparietal network 289 (Power et al., 2011) and used this system-level activity as an a priori brain measure to relate to 290 subsequent food cue activity.

$291 \quad$ Next, to estimate activity elicited by food cues when participants were watching 292 commercials, we ran a separate univariate GLM analysis modeling food events (i.e., whenever 293 featured food items appeared on the screen during a food commercial) and control (product) 294 events. Next, contrast images were calculated to compare activity when food events occurred 295 relative to that when control events occurred. Other commercial content (i.e., all time points 296 when products were not featured/visible on the screen) were modeled as baseline. We extracted 297 food cue related activity from these contrast images using independently defined, a priori 298 regions/systems of interest, namely: eight nodes of the frontoparietal control network, which 299 supports flexible exertion of self-control (Dosenbach et al., 2007; Power et al., 2011); and two 300 regions in the reward system, OFC and bilateral ventral striatum, which reliably activate to 
301 appetizing food images (MNI coordinates for OFC: $-30,33,-18$; VS: \pm 9, 3, -6; taken from

302 Wagner et al., 2013). We selected eight nodes in the frontoparietal control network to remain

303 consistent with our prior work showing that aggregate activity across these 8 nodes, versus

304 activity in a larger set of frontoparietal nodes, was most predictive of dieters' self-control success

305 and failure in daily life (Lopez et al., 2017). However, in order to investigate whether this effect

306 generalizes across a larger set of nodes we used 31 frontoparietal nodes from a recently

307 published parcellation study within our group based on a large, independent sample $(\mathrm{N}=828$;

308 Huckins et al., 2019) to re-compute regulation-reward balance scores. The findings presented in

309 the main text largely replicated (for more details see Supplementary Materials for all results from

310 this analysis). Food-cue specific activity in both control and reward regions was extracted using

311 a spherical ROI with a 6-millimeter radius.

312 For our main analysis, we related task-elicited activity in DLPFC and mean recruitment

313 of the frontoparietal network from the effortful self-control task to the relative recruitment of

314 frontoparietal control network (vs. reward system) during food cue exposure in the second fMRI

315 task. This relative recruitment was captured by regulation-reward balance scores, which were

316 calculated on a subject by subject basis by taking the difference of standardized (i.e., Z-scored),

317 averaged activity in the frontoparietal control network and activity in reward regions (i.e., OFC

318 and bilateral ventral striatum). Higher balance scores represent relatively greater recruitment of

319 the frontoparietal control network, whereas lower balance scores represent relatively greater

320 recruitment of reward regions; this procedure and operationalization of a brain-based balance

321 measure followed that of Lopez and colleagues' (2017) study.

322 The main data file used to run all models and compute all statistics is available at the

323 following repository on the Open Science Framework: https://osf.io/qpt3f/. 


\section{RESULTS}

325 First, as a manipulation check, we examined participants' ratings of their experience of

326 the first (effortful self-control) task they completed in the scanning session. On average,

327 participants found the task to be difficult, as inferred from a one-sample t-test against the scale's

328 midpoint value of $4, M=4.93$ (Difference from test value: $0.93,95 \%$ CI for difference: 0.45 ,

$3291.40), S D=1.21, t(26)=3.99, p<.001$, Cohen's $d=0.77$; this value was significantly greater

330 than dieters in a control condition from a similar study, $t(26)=3.09$, Cohen's $d=0.60, p=$

331.005 . The self-reported measures of difficulty and tiredness were not correlated with one another,

$332 r(25)=0.004, t=0.02, p=.985$, so they were entered in as separate covariates in multiple

333 regressions models described below. Next, we observed that across all 8 nodes of the FP

334 network, there was significant activity in the first fMRI task during effortful self-control (i.e.,

335 words versus film contrast), mean parameter estimate $=0.159(95 \% \mathrm{CI}: 0.092,0.226), \mathrm{SD}=$

$3360.169, t(26)=4.89$, Cohen's $d=0.941, p<.001$.

To relate brain activity during effortful self-control to subsequent food cue reactivity, we

first calculated the correlation between brain activity in this region and the relative balance of

activity in frontoparietal (vs. reward) regions during exposure to food commercials. There was a

significant negative association, such that those participants who more readily recruited the

DLPFC during the effortful self-control task had lower balance scores when viewing food

commercials, $r(24)=-0.574(95 \%$ bootstrapped CI with 10,000 iterations: $-0.753,-0.310), b=$ -

participants' self-reported difficulty and tiredness when they performed the effortful self-control task, as well as participants' body mass index (BMI), $b=-2.538(95 \% \mathrm{CI}:-4.08,-1.00), t(19)=-$

$3.445, p=.003$. In this multiple regression model, neither task difficulty $(b=-0.021, t(19)=$ - 
348 scores. Additionally, upon examining zero-order correlations, self-reported difficulty was not

349 associated with DLPFC activity during the effortful self-control task $(r=0.062, p=.760)$ or

350 balance scores during food cue reactivity $(r=-0.103, p=.615)$. This was also true for self-

351 reported tiredness, which did not correlate with DLPFC activity $(r=0.206, p=.302)$ or balance

352 scores $(r=-0.003, p=.988)$.

353 Next, consistent with the negative association between DLPFC activity during effortful

354 self-control and balance scores during subsequent exposure to food cues, there was also a

355 significant negative relationship between average recruitment of the frontoparietal network

356 activation during effortful self-control and balance scores in the cue reactivity task, $r(24)=$ -

3570.403 (95\% bootstrapped CI with 10,000 iterations: $-0.741,-0.032), b=-0.302, p=.041$, and

358 this relationship remained after controlling for self-reported difficulty and tiredness and BMI, $b=$

$359-0.339(95 \% \mathrm{CI}:-0.67,-0.01), t(19)=-2.168, p=.043$. Critically, the association between

360 frontoparietal network recruitment during effortful self-control was only observed with balance

361 scores during food cue-exposure. Specifically, there was no relationship between FP network

362 activity during self-control and subsequent FP network activity during cue-reactivity, $r(24)=$ -

$3630.206, t=-1.03, p=.313$, nor was initial FP network recruitment associated with reward system

364 activity during cue reactivity, $r(24)=-0.081, t=0.40, p=.693$. To rule out influences of

365 nuisance variables (e.g., differences in global BOLD signal between individuals) we extracted

366 parameter estimates based on an anatomical mask of the hippocampus (a region not engaged by

367 either task) and found no relationship during the first self-control task and balance scores in the

368 cue reactivity task, $r(24)=-0.09, t=-0.444, p=.661$.

369 Finally, we examined links between activity in the effortful self-control/cue reactivity

370 tasks and ad libitum ice-cream eating from the behavioral session. On average, participants 
371 consumed 71.1 grams of ice-cream ( $S D=40.8$, range: 9-158 grams), and there was a positive

372 (albeit non-significant) correlation between recruitment of DLPFC during effortful self-control

373 and grams of ice-cream consumed, $r(24)=0.319, t=1.647, p=.112$. There was also a negative,

374 non-significant relationship between balance scores and grams of ice-cream consumed, $r(24)=-$

$3750.309, t=-1.594, p=.124$.

\section{DISCUSSION}

377

378

379

380

381

382

383

384

385

386

387

388

389

390

391

392

393

Results from this study indicated that individual differences in chronic dieters' recruitment of brain regions associated with cognitive control (i.e., DLPFC and frontoparietal network) while performing an effortful self-control task was subsequently associated with the balance of activity between brain regions implicated in self-control and reward during exposure to food commercials. Consistent with our hypothesis, greater recruitment of DLPFC—as well as the frontoparietal network as a whole — during effortful self-control was associated with less recruitment of the frontoparietal network and more reward related activity whenever food items appeared on the screen in the cue reactivity task. This suggests that more (or less) brain activity during an initial task requiring self-control exertion may serve to characterize the different response profiles that dieters may show when exposed to appetitive food cues.

Notably, we only observed a significant, negative relationship between FP recruitment during effortful self-control and subsequent balance scores during exposure to food commercials. There was no such relationship between control-related FP recruitment in the first task and activity in either FP or reward systems, respectively, during food cue reactivity (second task).

This is consistent with theorizing that suggests taking both impulsive and inhibitory processes into account to characterize self-control outcomes (James, 1890; Kotabe \& Hofmann, 2015; Lewin, 1951), as well as recent evidence showing that the relative balance of control (versus 
394 reward) activity was predictive of self-regulation outcomes — whereas activity in either system 395 alone was not (Lopez et al., 2017).

The present findings also substantiate and extend previous studies that have shown that

397 398

400

401 402 403 404 405 406 407 408 409

410

411

412

413

414 415

when participants are engaged in effortful self-control tasks they subsequently show reduced brain activity in lateral prefrontal cortex when performing cognitive tasks (Friese et al., 2013; e.g., Luethi et al., 2016). For example, in a study by Friese and colleagues (2013), those participants who were randomly assigned to suppress their emotions when viewing highly distressing, negatively-valenced stimuli showed reduced recruitment of lateral prefrontal cortex during a subsequent Stroop task, compared to those participants in the control condition (Friese et al., 2013). However, one of the features that differentiates our study from previous work is that we focused on dieters' neural responses to appetitive food cues, following previous self-control exertion. This approach has the advantage of looking at a motivationally relevant class of stimuli in a population that, by definition, is chronically engaged in self-regulation and inherently motivated to regulate their responses. However, we would be remiss if we did not acknowledge that it remains an open question as to whether the effect of prior self-control exertion on neural responses to food cues reflects either: (1) a reduction in self-control capacity, as a resource-based account would predict (e.g., Baumeister, Muraven, \& Tice, 2000); or (2) the fact the those participants who experience the task as more effortful are simply less motivated to continue regulating in a secondary task, as per motivation-based accounts (e.g., Inzlicht \& Schmeichel, 2012).Without additional measures, the present study cannot disentangle these two views (see above, with associated references, for the most recent theorizing and findings related to potential mechanisms underlying self-control fatigue and lapses). 
eating of ice cream following a diet breaking episode in a separate experiment. Although the relationships were in the expected direction (i.e., greater grams of ice cream eaten among

419 participants who, on average, showed more FP recruitment during effortful self-control and also

420 lower balance scores), the overall correlations with neural measures were not significant.

421 Whether this reflects a true null effect, or instead that the mechanisms underlying individual

422 differences in neural activity we observed following self-control exertion do not translate to 423 disinhibited eating following a diet breaking episode, remains an open question.

There are several strengths to our study design and approach. First, our overall analysis approach—linking brain activity from an effortful self-control task to activity in a subsequent task that also calls for spontaneous regulation of prepotent responses - is arguably more ecologically valid than previous studies that have examined effortful self-control. Although speculative, we would argue that the sequence and nature of the two self-control tasks used here (i.e., an intensive, initial exertion of effort to inhibit, followed by unpredictable (but no less needed) instances that also require self-control) may mimic dieters' experiences in daily life. For example, a dieter might exert self-control to block out distraction during an intense time of study or work. And, the extent to which they find this sustained inhibition taxing, they may have little self-control capacity to call upon when they are suddenly faced with a dessert tray at a restaurant later that day.

Second, the current study's design may reliably identify those individuals who experience more (or less) success adhering to their dieting goals. For example, a dieter who more readily

437 recruits certain cognitive control regions (i.e., DLPFC) during an effortful self-control task may 438 be actively maintaining the task set (i.e., "avoid reading the words"), but such task engagement 
439 may be more cognitively demanding and render the frontoparietal network less able to exert

440 control over the reward system during future exposure to food cues (cf. Heatherton \& Wagner,

441 2011). Indeed, the effects observed here in the appetitive domain are consistent with other

442 studies that have showed reductions in activity in prefrontal cortex - and accompanying task

443 deficits in various cognitive tasks - following self-control exertion (Friese et al., 2013; Luethi et

444 al., 2016; e.g., Persson et al., 2013). The present findings are also consistent with predictions

445 made by models emphasizing the role of self-regulatory fatigue in affecting self-control

446 outcomes (Evans, Boggero, \& Segerstrom, 2015; Hofmann et al., 2012; Wilkowski et al., 2018),

447 and those that highlight the limitations of cognitive control processes more broadly (Shenhav et

448 al., 2017).

449 Additionally, if it is true that greater recruitment of brain regions during an effortful self450 control task can undermine future self-control attempts, then it means that more control-related

451 activity (during initial exertion) is not necessarily conducive to dieters' self-regulatory goals.

452 Indeed, and consistent with prior behavioral work on limited self-regulatory capacity (e.g.,

453 Baumeister et al., 1998), control-related activity during self-control exertion may reflect task

454 difficulty or effort, potentially leading to fatigue and dieters' inability to later recruit self-control

455 regions when faced with (subsequent) appetitive temptations. But, since participants' self-

456 reported difficulty/fatigue ratings did not correlate with brain activity during effortful self-

457 control, or with the frontoparietal (vs. reward) balance measure, it is possible that dieters do not

458 have conscious awareness or insight into how much they are affected by exertions of self-control

459 (or not). Future work may consider improving dieters' self-awareness about their susceptibility,

460 neural or otherwise, to improve adherence to self-regulatory goals over time. Indeed, others have 
461 made similar arguments about targeting people's self-awareness and insight in the context 462 treatments for drug addiction (Goldstein et al., 2009). worth mentioning. First, the self-control task we administered was somewhat short in length (i.e., 7 minutes), so it is not clear how long effects of effortful self-control on cue reactivity might persist. Second, even though our participants gave ratings of difficulty and fatigue during the effortful self-control task, we did not have an independent measure of participants' objective performance on the task (i.e., how successful they were in avoiding reading the words on the screen). A future study would benefit from incorporating eye-tracking or another validation measure to calculate, on a subject-by-subject basis, successful inhibition of the impulse to read the words. And even though we used a validated diet-breaking procedure for the behavioral session, using a self-control exertion task would have led to more construct validity and may

473 help explain why we did not find a relationship between the brain data and overeating after the 474 milkshake preload.

We should also note that, in comparison to behavioral work, the sample size was relatively small and thus was not powered for the detection of moderate correlations. This may

477 place a limit on our ability to detect small to moderate effects of self-control exertion (e.g., we 478 did not observe any relationship between brain activity and ice-cream eating). Although to 479 remain consistent with prior work, with recruitment necessarily constrained to female dieters, 480 future work would benefit from either expanding beyond this population in order to increase 481 sample sizes and test generalizability to other populations. Another possibility is that our sample, on average, showed relatively little ice cream consumption (mean $=71.1$ grams), as well as a more restricted range $(S D=40.8$ grams $)$ 
484 compared to previous studies that also measured disinhibited ice-cream eating in dieters (cf. 485 mean values of 71.7-211.2 grams and $S D$ values of 57.4-123.8 in Vohs \& Heatherton, 2000). 486 Also, in order to establish equivalent levels of motivations to regulate eating, we only recruited 487 from the dieting population. So, the boundary conditions and generalizability of this effect need 488 to be tested in other populations, as it is probable that the nature of self-regulatory goals and 489 other related factors may modulate patterns of brain activity during effortful self-control (and 490 subsequent food cue reactivity). Lastly, one overall caveat to the present work is that all reported 491 findings are correlational, so no strong claims can be made as far as the directionality of the 492 observed effects.

\section{CONCLUSIONS}

494 To conclude, we probed the neural mechanisms of repeated self-control exertion among 495 dieters, using a reasonably naturalistic dual-task paradigm that coupled an initial, effortful self496 control task with subsequent exposure to appetitive food cues as presented in real-world food 497 commercials. We extended past work, which took a brain-as-predictor approach (Berkman \& 498 Falk, 2013) using the balance between prefrontal and reward related responses during food cue 499 exposure to predict dietary failure (Lopez et al., 2014), by using this same measure to examine 500 how prior exertion of self-control may lead to impaired dietary self-control during exposure to 501 tempting food cues. In doing so, we tried to adopt a health neuroscience framework, a primary 502 aim of which is to better characterize health risk behaviors - including patterns of over-eating 503 that lead to obesity — in order to alleviate the various burdens, medical and otherwise, associated 504 with preventable chronic disease (Hall, Bickel, Erickson, \& Wagner, 2018). Future intervention 505 studies and clinical trials may benefit from taking a similar approach and target such brain-based 506 risk factor, with the goal of modulating brain responses during effortful self-control and cue 507 reactivity, and possibly producing changes in real world eating behaviors. 
508

509

510

511

512

513

514

515

516

517

518

519

520

521

522

523

524

525

526

527

528

529

530

531

532

533

534

535

536

537

538

539

540

541

542

543

544

545

546

547

548

549

550

551

\title{
ACKNOWLEDGEMENTS
}

\author{
None to declare.
}

\section{REFERENCES}

Aron, A. R., Robbins, T. W., \& Poldrack, R. A. (2014). Inhibition and the right inferior frontal cortex: one decade on. Trends in Cognitive Sciences, 18(4), 177-185.

Baumeister, R. F., Bratslavsky, E., Muraven, M., \& Tice, D. M. (1998). Ego depletion: is the active self a limited resource? Journal of Personality and Social Psychology, 74(5), 12521265.

Baumeister, R. F., Muraven, M., \& Tice, D. M. (2000). Ego Depletion: A Resource Model of Volition, Self-Regulation, and Controlled Processing. Social Cognition, 18(2), 130-150.

Baumeister, R. F., Tice, D. M., \& Vohs, K. D. (2018). The Strength Model of Self-Regulation: Conclusions From the Second Decade of Willpower Research. Perspectives on Psychological Science: A Journal of the Association for Psychological Science, 13(2), 141145.

Baumeister, R. F., Vohs, K. D., \& Tice, D. M. (2007). The Strength Model of Self-Control. Current Directions in Psychological Science, 16(6), 351-355.

Berkman, E. T., \& Falk, E. B. (2013). Beyond Brain Mapping: Using Neural Measures to Predict Real-World Outcomes. Current Directions in Psychological Science, 22(1), 45-50.

Berkman, E. T., Falk, E. B., \& Lieberman, M. D. (2011). In the trenches of real-world selfcontrol: neural correlates of breaking the link between craving and smoking. Psychological Science, 22(4), 498-506.

Berkman, E. T., Kahn, L. E., \& Merchant, J. S. (2014). Training-induced changes in inhibitory control network activity. The Journal of Neuroscience: The Official Journal of the Society for Neuroscience, 34(1), 149-157.

Brace, A., Crombag, H. S., \& Yeomans, M. R. (2016). Dissociating the effects of hedonic value and perceived energy content in a milkshake preload on subsequent behavioural impulsivity. Appetite, 101, 229.

Carver, C. S. (2005). Impulse and Constraint: Perspectives From Personality Psychology, Convergence With Theory in Other Areas, and Potential for Integration. Personality and Social Psychology Review: An Official Journal of the Society for Personality and Social Psychology, Inc, 9(4), 312-333.

Clarkson, J. J., Hirt, E. R., Jia, L., \& Alexander, M. B. (2010). When perception is more than reality: the effects of perceived versus actual resource depletion on self-regulatory behavior. Journal of Personality and Social Psychology, 98(1), 29-46.

Courtney, A. L., Rapuano, K. M., Sargent, J. D., Heatherton, T. F., \& Kelley, W. M. (2018). Reward System Activation in Response to Alcohol Advertisements Predicts College Drinking. Journal of Studies on Alcohol and Drugs, 79(1), 29-38.

Cox, R. W., Chen, G., Glen, D. R., Reynolds, R. C., \& Taylor, P. A. (2017). FMRI Clustering in AFNI: False-Positive Rates Redux. Brain Connectivity, 7(3), 152-171.

Demos, K. E., Heatherton, T. F., \& Kelley, W. M. (2012). Individual Differences in Nucleus Accumbens Activity to Food and Sexual Images Predict Weight Gain and Sexual Behavior. The Journal of Neuroscience: The Official Journal of the Society for Neuroscience, 32(16), 
552

553

554

555

556

557

558

559

560

561

562

563

564

565

566

567

568

569

570

571

572

573

574

575

576

577

578

579

580

581

582

583

584

585

586

587

588

589

590

591

592

593

594

595

596

597

5549-5552.

Demos, K. E., Kelley, W. M., \& Heatherton, T. F. (2011). Dietary restraint violations influence reward responses in nucleus accumbens and amygdala. Journal of Cognitive Neuroscience, 23(8), 1952-1963.

Dunst, B., Benedek, M., Jauk, E., Bergner, S., Koschutnig, K., Sommer, M., ... Neubauer, A. C. (2014). Neural efficiency as a function of task demands. Intelligence, 42(100), 22-30.

Evans, D. R., Boggero, I. A., \& Segerstrom, S. C. (2015). The Nature of Self-Regulatory Fatigue and "Ego Depletion": Lessons From Physical Fatigue. Personality and Social Psychology Review: An Official Journal of the Society for Personality and Social Psychology, Inc. Retrieved from http://eutils.ncbi.nlm.nih.gov/entrez/eutils/elink.fcgi?dbfrom=pubmed\&id=26228914\&retm ode $=$ ref\&cmd $=$ prlinks

Freeman, S. M., \& Aron, A. R. (2016). Withholding a Reward-driven Action: Studies of the Rise and Fall of Motor Activation and the Effect of Cognitive Depletion. Journal of Cognitive Neuroscience, 28(2), 237-251.

Friese, M., Binder, J., Luechinger, R., Boesiger, P., \& Rasch, B. (2013). Suppressing emotions impairs subsequent stroop performance and reduces prefrontal brain activation. PloS One, $8(4)$, e 60385.

Friese, M., Engeler, M., \& Florack, A. (2015). Self-perceived successful weight regulators are less affected by self-regulatory depletion in the domain of eating behavior. Eating Behaviors, 16, 5-8.

Friese, M., Loschelder, D. D., Gieseler, K., Frankenbach, J., \& Inzlicht, M. (2018). Is Ego Depletion Real? An Analysis of Arguments. Personality and Social Psychology Review: An Official Journal of the Society for Personality and Social Psychology, Inc, 1088868318762183.

Garrison, K. E., Finley, A. J., \& Schmeichel, B. J. (2018). Ego Depletion Reduces Attention Control: Evidence from Two High-Powered Preregistered Experiments. Personality \& Social Psychology Bulletin.

Goldstein, R. Z., Craig, A. D. B., Bechara, A., Garavan, H., Childress, A. R., Paulus, M. P., \& Volkow, N. D. (2009). The neurocircuitry of impaired insight in drug addiction. Trends in Cognitive Sciences, 13(9), 372-380.

Gray, J. R., Burgess, G. C., Schaefer, A., Yarkoni, T., Larsen, R. J., \& Braver, T. S. (2005). Affective personality differences in neural processing efficiency confirmed using fMRI. Cognitive, Affective \& Behavioral Neuroscience, 5(2), 182-190.

Haber, S. N., \& Knutson, B. (2010). The reward circuit: linking primate anatomy and human imaging. Neuropsychopharmacology: Official Publication of the American College of Neuropsychopharmacology, 35(1), 4-26.

Hagger, M. S., Chatzisarantis, N. L. D., Alberts, H., Anggono, C. O., Batailler, C., Birt, A. R., ... Zwienenberg, M. (2016). A Multilab Preregistered Replication of the Ego-Depletion Effect. Perspectives on Psychological Science: A Journal of the Association for Psychological Science, 11(4), 546-573.

Hall, P. A., Bickel, W. K., Erickson, K. I., \& Wagner, D. D. (2018). Neuroimaging, neuromodulation, and population health: the neuroscience of chronic disease prevention. Annals of the New York Academy of Sciences. https://doi.org/10.1111/nyas.13868

Heatherton, T. F., \& Baumeister, R. F. (1991). Binge eating as escape from self-awareness. Psychological Bulletin, 110(1), 86-108. 
598 Heatherton, T. F., Herman, C. P., Polivy, J., King, G. A., \& McGree, S. T. (1988). The

599

600

601

602

603

604

605

606

607

608

609

610

611

612

613

614

615

616

617

618

619

620

621

622

623

624

625

626

627

628

629

630

631

632

633

634

635

636

637

638

639

640

641

642

643 (mis)measurement of restraint: An analysis of conceptual and psychometric issues. Journal of Abnormal Psychology, 97(1), 19-28.

Heatherton, T. F., Polivy, J., \& Herman, C. P. (1991). Restraint, weight loss, and variability of body weight. Journal of Abnormal Psychology, 100(1), 78-83.

Heatherton, T. F., \& Wagner, D. D. (2011). Cognitive neuroscience of self-regulation failure. Trends in Cognitive Sciences, 15(3), 132-139.

Hedgcock, W. M., Vohs, K. D., \& Rao, A. R. (2012). Reducing self-control depletion effects through enhanced sensitivity to implementation: Evidence from fMRI and behavioral studies. Journal of Consumer Psychology: The Official Journal of the Society for Consumer Psychology, 22(4), 486-495.

Herman, C. P., \& Mack, D. (1975). Restrained and unrestrained eating. Journal of Personality, 43(4), 647-660.

Herman, C. P., \& Polivy, J. (1980). Restrained eating. In A. J. Stunkard (Ed.), Obesity (pp. 208225). Saunders.

Herman, C. P., Polivy, J., \& Esses, V. M. (1987). The illusion of counter-regulation. Appetite, 9(3), 161-169.

Hofmann, W., Baumeister, R. F., Förster, G., \& Vohs, K. D. (2012). Everyday temptations: an experience sampling study of desire, conflict, and self-control. Journal of Personality and Social Psychology, 102(6), 1318-1335.

Hofmann, W., Friese, M., \& Strack, F. (2009). Impulse and Self-Control From a Dual-Systems Perspective. Perspectives on Psychological Science: A Journal of the Association for Psychological Science, 4(2), 162-176.

Huckins, J. F., Adeyemo, B., Miezin, F. M., Power, J. D., Gordon, E. M., Laumann, T. O., ... \& Kelley, W. M. (2019). Reward-related regions form a preferentially coupled system at rest. Human Brain Mapping, 40(2), 361-376.

Inzlicht, M., \& Schmeichel, B. J. (2012). What Is Ego Depletion? Toward a Mechanistic Revision of the Resource Model of Self-Control. Perspectives on Psychological Science: A Journal of the Association for Psychological Science, 7(5), 450-463.

Inzlicht, M., Schmeichel, B. J., \& Macrae, C. N. (2014). Why self-control seems (but may not be) limited. Trends in Cognitive Sciences, 18(3), 127-133.

James, W. (1890). The Principles of Psychology (Vol. 2). Mineola, NY: Dover.

Job, V., Dweck, C. S., \& Walton, G. M. (2010). Ego depletion-Is it all in your head? Implicit theories about willpower affect self-regulation. Psychological Science. Retrieved from http://journals.sagepub.com/doi/abs/10.1177/0956797610384745

Kahan, D., Polivy, J., \& Herman, C. P. (2003). Conformity and dietary disinhibition: a test of the ego-strength model of self-regulation. The International Journal of Eating Disorders, 33(2), $165-171$.

Kelley, W. M., Wagner, D. D., \& Heatherton, T. F. (2015). In Search of a Human SelfRegulation System. Annual Review of Neuroscience, 38(1), 389-411.

Kotabe, H. P., \& Hofmann, W. (2015). On Integrating the Components of Self-Control. Perspectives on Psychological Science: A Journal of the Association for Psychological Science, 10(5), 618-638.

Lewin, K. (1951). Field Theory in Social Science: Selected Theoretical Papers. Harper.

Lopez, R. B., Chen, P.-H. A., Huckins, J. F., Hofmann, W., Kelley, W. M., \& Heatherton, T. F. (2017). A balance of activity in brain control and reward systems predicts self-regulatory 
644

645

646

647

648

649

650

651

652

653

654

655

656

657

658

659

660

661

662

663

664

665

666

667

668

669

670

671

672

673

674

675

676

677

678

679

680

681

682

683

684

685

686

687

688

689 outcomes. Social Cognitive and Affective Neuroscience, 12(5), 832-838.

Lopez, R. B., Hofmann, W., Wagner, D. D., Kelley, W. M., \& Heatherton, T. F. (2014). Neural Predictors of Giving in to Temptation in Daily Life. Psychological Science, 25(7), 13371344.

Lopez, R. B., Milyavskaya, M., Hofmann, W., \& Heatherton, T. F. (2016). Motivational and neural correlates of self-control of eating: a combined neuroimaging and experience sampling study in dieting female college students. Appetite, 103, 192-199.

Lowe, M. R., Annunziato, R. A., Markowitz, J. T., Didie, E., Bellace, D. L., Riddell, L., ... Stice, E. (2006). Multiple types of dieting prospectively predict weight gain during the freshman year of college. Appetite, 47(1), 83-90.

Lowe, M. R., Doshi, S. D., Katterman, S. N., \& Feig, E. H. (2013). Dieting and restrained eating as prospective predictors of weight gain. Frontiers in Psychology, 4, 577.

Luethi, M. S., Friese, M., Binder, J., Boesiger, P., Luechinger, R., \& Rasch, B. (2016). Motivational incentives lead to a strong increase in lateral prefrontal activity after selfcontrol exertion. Social Cognitive and Affective Neuroscience, 11(10), 1618-1626.

Lurquin, J. H., \& Miyake, A. (2017). Challenges to Ego-Depletion Research Go beyond the Replication Crisis: A Need for Tackling the Conceptual Crisis. Frontiers in Psychology, 8, 568.

Mills, J. S., \& Palandra, A. (2008). Perceived caloric content of a preload and disinhibition among restrained eaters. Appetite, 50(2-3), 240-245.

Muraven, M., Tice, D. M., \& Baumeister, R. F. (1998). Self-control as a limited resource: Regulatory depletion patterns. Journal of Personality and Social Psychology, 74(3), 774.

O'Doherty, J. P. (2004). Reward representations and reward-related learning in the human brain: insights from neuroimaging. Current Opinion in Neurobiology, 14(6), 769-776.

Persson, J., Larsson, A., \& Reuter-Lorenz, P. A. (2013). Imaging fatigue of interference control reveals the neural basis of executive resource depletion. Journal of Cognitive Neuroscience, 25(3), 338-351.

Petersen, S. E., van Mier, H., Fiez, J. A., \& Raichle, M. E. (1998). The effects of practice on the functional anatomy of task performance. Proceedings of the National Academy of Sciences of the United States of America, 95(3), 853-860.

Power, J. D., Cohen, A. L., Nelson, S. M., Wig, G. S., Barnes, K. A., Church, J. A., ... Petersen, S. E. (2011). Functional Network Organization of the Human Brain. Neuron, 72(4), 665678.

Rapuano, K. M., Huckins, J. F., Sargent, J. D., Heatherton, T. F., \& Kelley, W. M. (2015). Individual Differences in Reward and Somatosensory-Motor Brain Regions Correlate with Adiposity in Adolescents. Cerebral Cortex , 26(6), 2602-2611.

Rapuano, K. M., Zieselman, A. L., Kelley, W. M., Sargent, J. D., Heatherton, T. F., \& GilbertDiamond, D. (2016). Genetic risk for obesity predicts nucleus accumbens size and responsivity to real-world food cues. Proceedings of the National Academy of Sciences of the United States of America, 114(1), 160-165.

Sayala, S., Sala, J. B., \& Courtney, S. M. (2006). Increased neural efficiency with repeated performance of a working memory task is information-type dependent. Cerebral Cortex, 16(5), 609-617.

Shenhav, A., Musslick, S., Lieder, F., Kool, W., Griffiths, T. L., Cohen, J. D., \& Botvinick, M. M. (2017). Toward a Rational and Mechanistic Account of Mental Effort. Annual Review of Neuroscience, 40, 99-124. 
690 Suzuki, S., Cross, L., \& O’Doherty, J. P. (2017). Elucidating the underlying components of food

691

692

693

694

695

696

697

698

699

700

701

702

703

704

705 valuation in the human orbitofrontal cortex. Nature Neuroscience, 20(12), 1780-1786.

Timko, C. A., Juarascio, A., \& Chowansky, A. (2012). The effect of a pre-load experiment on subsequent food consumption. Caloric and macronutrient intake in the days following a preload manipulation. Appetite, 58(2), 747-753.

Tomiyama, A. J., Moskovich, A., Haltom, K. B., Ju, T., \& Mann, T. (2009). Consumption after a diet violation: disinhibition or compensation? Psychological Science, 20(10), 1275-1281.

Vohs, K. D., \& Heatherton, T. F. (2000). Self-regulatory failure: a resource-depletion approach. Psychological Science, 11(3), 249-254.

Wagner, D. D., Altman, M., Boswell, R. G., Kelley, W. M., \& Heatherton, T. F. (2013). SelfRegulatory Depletion Enhances Neural Responses to Rewards and Impairs Top-Down Control. Psychological Science, 24(11), 2262-2271.

Wilkowski, B. M., Ferguson, E. L., Williamson, L. Z., \& Lappi, S. K. (2018). (How) Does Initial Self-Control Undermine Later Self-Control in Daily Life? Personality \& Social Psychology Bulletin, 146167218766857. 


\section{Table $\mathbf{1}$ (on next page)}

Supra-threshold brain regions activated during effortful self-control from the first fMRI task. 
1 Table 1. Supra-threshold brain regions activated during effortful self-control from the first fMRI 2 task.

3

\begin{tabular}{|l|c|c|c|}
\hline \multicolumn{1}{|c|}{ Region* } & $\begin{array}{c}\text { MNI coordinates } \\
\text { of peak voxel }\end{array}$ & \# voxels & $\begin{array}{c}\text { Effect size } \\
(t \text { value })\end{array}$ \\
\hline Right dorsolateral prefrontal cortex & $30,42,24$ & 206 & 5.42 \\
\hline Right superior frontal gyrus & $21,-3,63$ & 2207 & 8.17 \\
\hline Right anterior insula/frontal operculum & $36,18,3$ & 329 & 7.09 \\
\hline Left superior parietal lobe & $-24,-54,57$ & 6788 & 13.14 \\
\hline Right cerebellum & $9,-78,-24$ & 192 & 7.29 \\
\hline Left anterior insula/frontal operculum & $-30,21,3$ & 111 & 7.03 \\
\hline Right occipital pole & $9,-90,9$ & 229 & 7.53 \\
\hline
\end{tabular}

4 *Unless otherwise indicated, labels are taken from the Harvard-Oxford cortical atlas (Desikan et al. 2006) 


\section{Figure 1}

Task-elicited brain activity related to effortful self-control, with eight nodes comprising the frontoparietal control network (Power et al., 2011) indicated by black spheres.
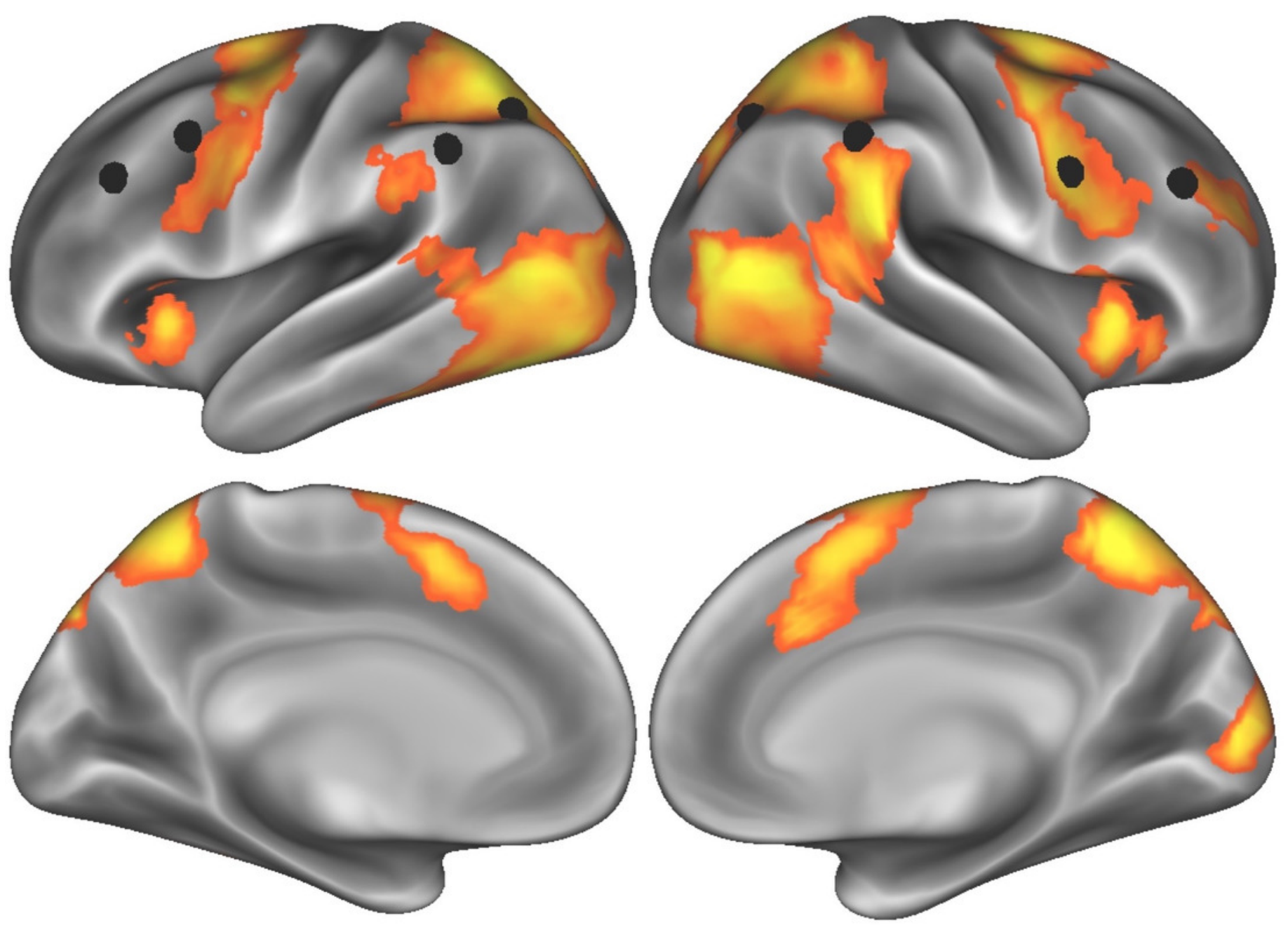
Figure 2

Negative association between DLPFC activity during effortful self-control and subsequent frontoparietal (vs. reward) balance scores during exposure to food commercials.

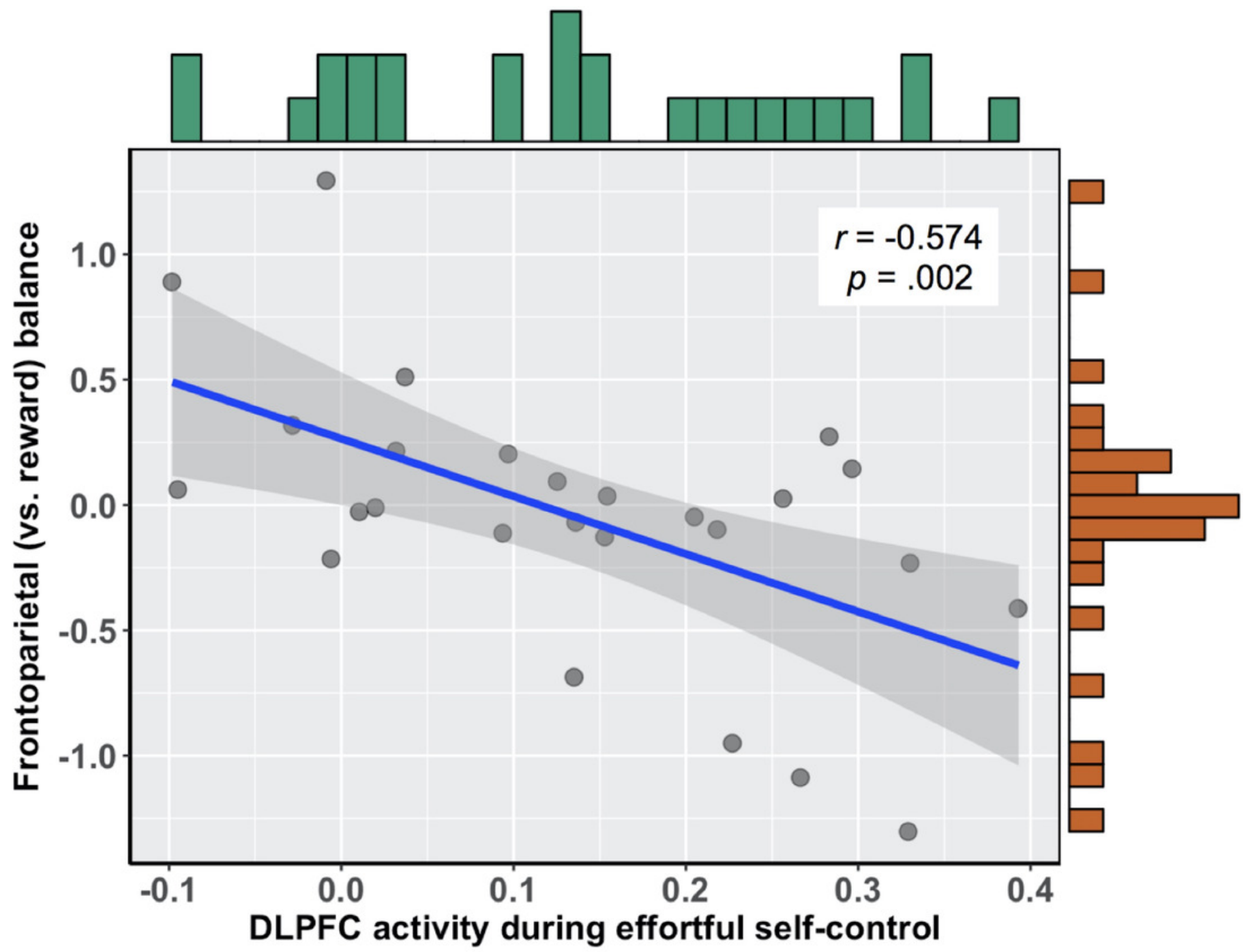

